\title{
A Local Controller for Discrete-Time Large-Scale System by Using Integral Variable Structure Control
}

\author{
C. H. Chai and Johari H. S. Osman \\ Faculty of Electrical Engineering, University Technology Malaysia, 81300 Skudai, Malaysia \\ Correspondence should be addressed to C. H. Chai; chai8987@gmail.com
}

Received 8 June 2015; Revised 5 October 2015; Accepted 5 October 2015

Academic Editor: Xiao He

Copyright ( 2015 C. H. Chai and J. H. S. Osman. This is an open access article distributed under the Creative Commons Attribution License, which permits unrestricted use, distribution, and reproduction in any medium, provided the original work is properly cited.

\begin{abstract}
A new local controller for discrete-time integral variable structure control of a large-scale system with matched and unmatched uncertainty is presented. The local controller is able to bring the large-scale system into stability by using only the states feedback from individual subsystem itself. A new theorem is established and proved that the controller is able to handle the effect of interconnection for the large-scale system with matched and unmatched uncertainty, and the system stability is ensured. The controller is able to control the system to achieve the quasi-sliding surface and remains on it. The results showed a fast convergence to the desired value and the attenuation of disturbance is achieved.
\end{abstract}

\section{Introduction}

As defined by Siljak [1], large-scale systems usually refer to systems that consist of a large number of state variables, system parametric uncertainties, a complex structure, and a strong interaction between subsystems. When the size of the dynamical system increases, the implementation of centralized control will be either impossible or uneconomical. Research in decentralized control has been motivated by the inadequacy of conventional modern control theory to deal with certain issues in large-scale system, such as the issue that it is impossible to incorporate so many feedback loops into the centralized design. Decentralized control theory has risen in response to the difficulty that there are restrictions on information transfer between certain groups of sensors or actuators [2]. In any case where there is a failure or insufficient information transfer between subsystems, local controller will play a significant role in controlling the largescale system. Chen [3] has developed a class of local control, utilizing solely the states of each individual subsystem and the bounds of the uncertainties that assured the desired properties when the proposed requirements on a test matrix are satisfied.
In the development of decentralized control on discretetime large-scale systems, Li et al. [4] have used decentralized control by dynamic programming method to achieve the control of three-reach river pollution problem. This paper addressed the dynamic issue of the interconnections and external disturbance of the systems. The challenge for this method is the speed of computation required for systems with fast respond. Lyou and Bien [5] used the adaptive feedback concept for stabilization of the interconnected system with uncertain system parameters and the feed-forward concept for compensation of bounded deterministic disturbances to control a large-scale interconnected discrete system. Subsequently, linear matrix inequality (LMI) technique has been used by Park and Lee [6] to derive a sufficient condition for robust stability in decentralized discrete-time large-scale systems with parametric uncertainty. Park et al. [7] applied the dynamic output feedback controller design to a discretetime large-scale system with delay at subsystem interconnections. Lyapunov method has been combined with LMI technique to develop the dynamic output feedback controller to guarantee the cost stabilization of the systems and achieve asymptotically stable closed-loop system with adequate level of performance. Ou et al. [8] also used LMI method to achieve 
the stability analysis and $H_{\infty}$ controller design to achieve disturbance attenuation performance by using Fuzzy Logic approach for the decentralized control of discrete-time largescale systems.

In the early development stage of discrete-time variable structure control (VSC) theory, the basic conditions for achieving the equivalent of sliding mode as in continuoustime variable structure control have been proposed by Dote and Hoft [9], Sarpturk et al. [10], Milosavljevic [11], and Furuta [12]. Method for quasi-sliding mode design and the use of reaching law approach to develop the control law for robust control in discrete-time VSC has been proposed by Gao et al. [13]. Discrete-time integral sliding mode control for sampled data system under state regulation was reported in Abidi et al. [14]. Subsequently, Xi and Hesketh [15] demonstrated the discrete-time integral sliding mode system to deal with both matched and unmatched uncertainties focused on SISO system.

A discrete-time large-scale system in VSC has been introduced by Sheta [16] with optimum control method. His study focused on the uncertain changes in the interconnection between subsystems and these uncertainties were governed by Markov chain technique. The controller was designed offline based on a set of expected system failure modes and switched on-line when failure was detected. Apart from this, literatures that focus on the research of discrete-time VSC for large-scale systems are rather limited.

Traditionally, the local control for a nonlinear plant by using linear systems theory is based upon linearization of the underlying nonlinear dynamics in the region of some operating points that correspond to a physical equilibrium of a plant. Thus the nonlinear system is treated as multiple time-varying linear systems that operate in a range of distinct equilibria, and gain scheduling approach was used to deal with the transition between equilibria. Other well-known methods were multiple model adaptive control and local controller network [17]. These methods usually have bigger challenge to control when the systems' uncertainty and disturbances kicked in, due to prior knowledge of the changes that are needed to generate the gain parameters. On the other hand, Markov jump large-scale system control by using local mode-dependent decentralized robust $H_{\infty}$ has been considered by Zhuansun and Xiong [18] to tackle the internal uncertainties of the local subsystems and interconnection uncertainties between subsystems. Markov method requires extensive computational resources especially when the system is large; thus it is a challenge for certain application that is fast changing and requires fast respond. VSC with the advantage in rejecting the uncertainty and disturbances to local subsystems and interconnection between subsystems is a better option in this case.

In this paper, a new theorem of using integral VSC method to establish a local controller for a large-scale discrete-time system with unmatched uncertainties is proposed. The controller only utilizes the states feedback from the individual subsystem without the information from other interconnected subsystems. The simulation results have shown that the proposed controller rendered the large-scale system to be stable and able to handle the effect of the interconnections and unmatched uncertainties. This local controller is very useful for controlling large-scale system with difficulty in obtaining the feedback from other subsystems and can only utilize the states feedback information from the local subsystems.

This paper is organized into 6 sections; Section 1 is the introduction and Section 2 introduces the preliminary concept of VSC, followed by the problem statement in Section 3. The controller design and proof of the theorem are given in Section 4. Section 5 presents the simulation results of a discrete-time large-scale system under study and the conclusion is given in Section 6.

\section{Preliminaries}

Consider a discrete-time system in the perturbed condition:

$$
x(k+1)=A x(k)+B u(k)+d(k),
$$

where $A$ represent system parameters, $B$ represent input parameters, and $d(k)$ is the disturbances.

The VSC requires that the integral sliding surface is designed such that the system response restricted to $\sigma(k+1)=$ $\sigma(k)=0$ has a desired behavior such as stability or tracking. The integral VSC sliding surface equation is given by

$$
\sigma(k)=G x(k)-G x(0)+h(k)
$$

with $h(k)$ being iteratively computed as $h(k)=h(k-1)-$ $G x(k-1)$

The VSC control input portion is then given by

$$
u(k)=-(G B)^{-1}(G A x(k)+G \widehat{d}(k)-G x(0)+h(k)),
$$

where $\widehat{d}(k)$ is the disturbance compensation.

\section{Problem Statement}

This paper considers a discrete-time large-scale system given by

$$
x(k+1)=A x(k)+B u(k)+d(k),
$$

where $A$ is the system parameter, $B$ is the input parameter, and $d(k)$ represent the system matched and unmatched uncertainty, disturbances, and interconnection between subsystems.

It is assumed that the system can be decomposed into $p$ subsystems as follows:

$$
\begin{aligned}
x_{i}(k+1)= & A_{i} x_{i}(k)+B_{i} u_{i}(k)+B_{i} f_{m i}(k)+f_{u i}(k) \\
& +V_{i} \sum_{\substack{j=1 \\
j \neq i}}^{p} \emptyset_{j i} z_{j i}(k), \\
x_{i}(k+1)= & A_{i} x_{i}(k)+B_{i}\left(u_{i}(k)+f_{m i}(k)\right) \\
& +V_{i} \sum_{\substack{j=1 \\
j \neq i}}^{p} \emptyset_{j i} z_{j i}(k)+f_{u i}(k), \\
& (i=1.2, \ldots, p),
\end{aligned}
$$


where $A_{i}$ is system parameter, $B_{i}$ is input parameter, $x_{i}(k+$ 1) $\in R^{n}, u_{i}(k) \in R^{m}, f_{m i}(k)$ is the matched uncertainty, $f_{u i}(k)$ is the unmatched uncertainty, $V_{i} \& \emptyset_{j i}$ is constant matrix with appropriate dimension, $z_{j i}(k) \in R^{q_{j}}$ is the interconnection between subsystem $j$ and $i$ with

$$
\begin{aligned}
z_{j i}(k+1) & =A_{z j i} z_{j i}(k)+\psi_{j i} y_{i}(k), \\
y_{i}(k) & =c_{i} x_{i}(k), \\
x_{i}(k) & =x_{i 0},
\end{aligned}
$$

$k=0, \emptyset_{j i}, \psi_{j i}$, and $A_{z j i}$ are matrix with appropriate dimension. that is,

It is assumed that both $f_{m i}(k)$ and $f_{u i}(k)$ are bounded;

$$
\begin{aligned}
& 0 \leq f_{m i}(k) \leq F_{m}, \\
& 0 \leq f_{u i}(k) \leq F_{u},
\end{aligned}
$$

and the bounds are known.

Assumption 1. $G_{i} B_{i}$ is invertible ( $G$ can be arbitrarily chosen by assuming that the following conditions are met), according to $\mathrm{Xi}$ and Hesketh [15]:

$$
\begin{aligned}
\left|G_{i} B_{i}\left(f_{m i}(k)-\widehat{f}_{m i}(k)\right)\right| & <N, \\
0 & <N<\infty, \\
\left|G_{i}\left(f_{u i}(k)-\widehat{f}_{u i}(k)\right)\right| & <M, \\
0 & <M<\infty, \\
\left|G_{i} V_{i}\left(\sum_{\substack{j=1 \\
j \neq i}}^{p} \emptyset_{j i} z_{j i}(k)-\sum_{\substack{j=1 \\
j \neq i}}^{p} \emptyset_{j i} z_{j i}(k-1)\right)\right| & <L, \\
\mid & 0<L<\infty .
\end{aligned}
$$

It is assumed that $N, M$, and $L$ are known, and $\widehat{f}_{m i}(k)$ and $\widehat{f}_{u i}(k)$ are the last value of disturbances.

According to Su et al. [19], the last value of a disturbance signal can be taken as estimation of its current value if the updated value is not accessible, under the assumption that the disturbance is continuous and smooth. As $\widehat{f}_{m i}(k)$ represents the last value measureable from the system, it is equivalent to the previous state, $f_{m i}(k-1)$ :

$$
\begin{aligned}
f_{m i}(k)-\widehat{f}_{m i}(k) & =f_{m i}(k)-f_{m i}(k-1), \\
f_{u i}(k)-\widehat{f}_{u i}(k) & =f_{u i}(k)-f_{u i}(k-1) .
\end{aligned}
$$

The idea here is to find an approximation that is part of the disturbance to estimate the past value of disturbance for the calculation to cancel the present disturbance. The approximation is good only for a small change in the disturbance between consecutive sampling periods that the sampling rate is high as compared to the frequency composition of the disturbance [20].

The objective is then to design a decentralized controller, $u_{i}(k)$, such that the large-scale discrete-time system (5) and (6) can be controlled.

\section{Integral Variable Structure Controller}

A decentralized discrete-time controller, $u_{i}(k)$, based on integral VSC technique is proposed for each subsystem. The sliding surface is designed for each subsystem followed by the switching controller as presented in the following.

In order to guarantee the existence of sliding mode and reduce chattering effect, the following condition must be satisfied [10]:

$$
\sigma_{i}(k)\left(\sigma_{i}(k+1)-\sigma_{i}(k)\right) \leq 0 \text {; }
$$

$$
\left|\sigma_{i}(k+1)\right| \leq\left|\sigma_{i}(k)\right|
$$

4.1. Sliding Surface Design. A discrete-time integral sliding surface for each subsystem is designed as

$$
\sigma_{i}(k)=G_{i} x_{i}(k)-G_{i} x_{i}(0)+h_{i}(k),
$$

where $h_{i}(k)$ is iteratively computed as

$$
\begin{aligned}
h_{i}(k)= & h_{i}(k-1) \\
& -\left(G_{i} B_{i} u_{i 0}(k-1)+G_{i} A_{i} x_{i}(k-1)\right)
\end{aligned}
$$

with $\sigma_{i}(k) \in R^{n}, h_{i}(k) \in R^{m}, h_{i}(0)=0$, and $G_{i} \in R^{1 \times n}$.

It is assumed that the control law is given as

$$
u_{i}(k)=u_{i 0}(k)+u_{i 1}(k)
$$

where the first component, $u_{i 0}(k)=-K_{i} x_{i}(k)$, is the equivalent control portion after system achieves the quasi-sliding mode. The gain $K$ is to be designed later.

By taking $h_{i}(k+1)=h_{i}(k)-\left(G_{i} B_{i} u_{i 0}(k)+G_{i} A_{i} x_{i}(k)\right)$, the sliding surface dynamics can be obtained from (5), (14), and (15) as follows:

$$
\begin{aligned}
& \sigma_{i}(k+1)=G_{i} x_{i}(k+1)-G_{i} x_{i}(0)+h_{i}(k+1) \\
& =G_{i}\left(A_{i} x_{i}(k)+B_{i}\left(u_{i}(k)+f_{m i}(k)\right)\right. \\
& \left.+V_{i} \sum_{\substack{j=1 \\
j \neq i}}^{p} \emptyset_{j i} z_{j i}(k)+f_{u i}(k)\right)-G_{i} x_{i}(0)+h_{i}(k)
\end{aligned}
$$




$$
\begin{aligned}
& -G_{i} B_{i} u_{i 0}(k)-G_{i} A_{i} x_{i}(k)=G_{i} A_{i} x_{i}(k) \\
& +G_{i} B_{i}\left(u_{i}(k)+f_{m i}(k)\right)+G_{i} f_{u i}(k) \\
& +G_{i} V_{i} \sum_{\substack{j=1 \\
j \neq i}}^{p} \emptyset_{j i} z_{j i}(k)-G_{i} x_{i}(0)+h_{i}(k)-G_{i} B_{i} u_{0}(k) \\
& -G_{i} A_{i} x_{i}(k) .
\end{aligned}
$$

By substituting (16) into (17), the equation of sliding surface becomes

$$
\begin{aligned}
\sigma_{i}(k+1)= & G_{i} B_{i} u_{i 1}(k)+G_{i} B_{i} f_{m i}(k)+G_{i} f_{u i}(k) \\
& +G_{i} V_{i} \sum_{\substack{j=1 \\
j \neq i}}^{p} \emptyset_{j i} z_{j i}(k)+h_{i}(k)-G_{i} x_{i}(0) .
\end{aligned}
$$

From (14) and (18),

$$
\begin{aligned}
\sigma_{i}(k+1)-\sigma_{i}(k)= & G_{i} B_{i} u_{i 1}(k)+G_{i} B_{i} f_{m i}(k) \\
& +G_{i} f_{u i}(k)+G_{i} V_{i} \sum_{\substack{j=1 \\
j \neq i}}^{p} \emptyset_{j i} z_{j i}(k) \\
& -G_{i} x_{i}(k) .
\end{aligned}
$$

4.2. Controller Design. The controller is designed according to (17) with two components, $u_{i}(k)=u_{i 0}(k)+u_{i 1}(k)$, where the second component is given as

$$
\begin{aligned}
& u_{i 1}(k)=-\left(G_{i} B_{i}\right)^{-1}\left[G_{i} B_{i} \widehat{f}_{m i}(k)+G_{i} \widehat{f}_{u i}(k)\right. \\
& \left.-G_{i} x_{i}(k)+\alpha_{i}(k) \sigma_{i}(k)+\beta_{i}(k) \operatorname{sgn}\left(\sigma_{i}(k)\right)\right],
\end{aligned}
$$

where

$$
\begin{aligned}
& \alpha_{i}(k)=0, \\
& \beta_{i}(k)=0
\end{aligned}
$$

$$
\text { when } \sigma_{i}(k)=0
$$

or

$$
\begin{aligned}
& \alpha_{i}(k)=\gamma_{i}(k) \operatorname{sgn}\left(\sigma_{i}(k)\right)+\epsilon_{i}, \\
& \text { with } \gamma_{i}(k)=\frac{N+M+L}{\left|\sigma_{i}(k)\right|}, 0<\epsilon_{i}<1, \\
& \beta_{i}(k)=\lambda_{i}\left|\left(1-\epsilon_{i}\right) \sigma_{i}(k)\right|, \quad 0<\lambda_{i}<1
\end{aligned}
$$

otherwise.

$u_{i 1}(k)$ is the reaching mode control component that will ensure that the system is able to achieve a quasi-sliding mode.

Theorem 2. Subject to Assumption 1 and sliding surface design of (14), the large-scale discrete-time system will achieve quasisliding mode and remain in it by having the control input of (16).
Proof. The proof of the theorem is given below.

While $\sigma_{i}(k) \neq 0$, define a Lyapunov function:

$$
J(k)=0.5 \sigma_{i}^{2}(k) .
$$

Ensuring that $J(k)$ is nonincreasing is equivalent to ensuring the condition in (12) and (13) [15].

Let

$$
\begin{aligned}
Q_{i}(k)= & G_{i} B_{i}\left(f_{m i}(k)-\widehat{f}_{m i}(k)\right) \\
& +G_{i}\left(f_{u i}(k)-\widehat{f}_{u i}(k)\right)+G_{i} V_{i} \sum_{\substack{j=1 \\
j \neq i}}^{p} \emptyset_{j i} z_{j i}(k) .
\end{aligned}
$$

Substituting (23) into (20) gives

$$
\begin{aligned}
\sigma_{i}(k+1)-\sigma_{i}(k)= & -\alpha_{i}(k) \sigma_{i}(k)-\beta_{i}(k) \operatorname{sgn}\left(\sigma_{i}(k)\right) \\
& +G_{i} B_{i}\left(f_{m i}(k)-\widehat{f}_{m i}(k)\right) \\
& +G_{i}\left(f_{u i}(k)-\widehat{f}_{u i}(k)\right) \\
& +G_{i} V_{i} \sum_{\substack{j=1 \\
j \neq i}}^{p} \emptyset_{j i} z_{j i}(k) .
\end{aligned}
$$

Multiplying both sides with $\sigma_{i}(k)$ gives

$$
\begin{aligned}
\sigma_{i}(k) & \left(\sigma_{i}(k+1)-\sigma_{i}(k)\right) \\
= & -\alpha_{i}(k) \sigma_{i}^{2}(k)-\beta_{i}(k) \sigma_{i}(k) \operatorname{sgn}\left(\sigma_{i}(k)\right) \\
& +Q_{i}(k) \sigma_{i}(k) \\
= & \sigma_{i}(k)\left(Q_{i}(k)-\alpha_{i}(k) \sigma_{i}(k)\right)-\beta_{i}(k)\left|\sigma_{i}(k)\right| .
\end{aligned}
$$

When $\sigma_{i}(k)<0$, this implies that

$$
\sigma_{i}(k)\left(\sigma_{i}(k+1)-\sigma_{i}(k)\right)<0, \quad \text { provided } \alpha_{i}(k)>0 .
$$

Subsequently, following the conditions stated in (10), it can be shown that

$$
Q_{i}(k)<\left\|Q_{i}(k)\right\|<M+N+L=\gamma_{i}(k)\left|\sigma_{i}(k)\right| .
$$

When $\sigma_{i}(k)>0$ and since $\gamma_{i}(k) \sigma_{i}^{2}(k)>Q_{i}(k) \sigma_{i}(k),(26)$ becomes

$$
\begin{aligned}
\left(\sigma_{i}(k+1)-\sigma_{i}(k)\right) & \\
< & \left(\gamma_{i}(k)-\alpha_{i}(k)\right) \sigma_{i}^{2}(k)-\beta_{i}(k)\left|\sigma_{i}(k)\right| \\
= & \left(\gamma_{i}(k) \operatorname{sgn}\left(\sigma_{i}(k)\right)-\alpha_{i}(k)\right) \sigma_{i}^{2}(k) \\
& \quad-\beta_{i}(k)\left|\sigma_{i}(k)\right|=-\epsilon_{i} \sigma_{i}^{2}(k)-\beta_{i}(k)\left|\sigma_{i}(k)\right| \leq 0 .
\end{aligned}
$$

Substituting $Q_{i}(k)$ as defined in (24) into (25) gives

$$
\begin{aligned}
\sigma_{i}(k+1)= & \left(\sigma_{i}(k)+Q_{i}(k)-\alpha_{i}(k) \sigma_{i}(k)\right) \\
& -\beta_{i}(k) \operatorname{sgn}\left(\sigma_{i}(k)\right) .
\end{aligned}
$$


Since it is defined in (20) that $\gamma_{i}(k)=(N+M+L) /\left|\sigma_{i}(k)\right|$ and $\alpha_{i}(k)=\gamma_{i}(k) \operatorname{sgn}\left(\sigma_{i}(k)\right)+\epsilon_{i}$, let $\varsigma_{i}=\gamma_{i}(k)\left|\sigma_{i}(k)\right|-Q_{i}(k)=M+$ $N+L-Q_{i}(k)$, and it is known that $\left|\sigma_{i}(k)\right|=\sigma_{i}(k) \operatorname{sgn}\left(\sigma_{i}(k)\right)$ according to [1], substitute $Q_{i}(k)=\gamma_{i}(k)\left|\sigma_{i}(k)\right|-\varsigma_{i}$ and $\epsilon_{i}=$ $\gamma_{i}(k) \operatorname{sgn}\left(\sigma_{i}(k)\right)-\alpha_{i}(k)$ into equation below:

$$
\begin{aligned}
& \left|\sigma_{i}(k+1)\right|=\mid\left(\sigma_{i}(k)+Q_{i}(k)-\alpha_{i}(k) \sigma_{i}(k)\right) \\
& \quad-\beta_{i}(k) \operatorname{sgn}\left(\sigma_{i}(k)\right)|=|\left(1-\alpha_{i}(k)\right) \sigma_{i}(k) \\
& \quad+\left(\gamma_{i}(k)\left|\sigma_{i}(k)\right|-\varsigma_{i}\right)-\beta_{i}(k) \operatorname{sgn}\left(\sigma_{i}(k)\right) \mid \\
& \quad=\mid\left(1-\alpha_{i}(k)\right) \sigma_{i}(k) \\
& \quad+\left(\gamma_{i}(k) \operatorname{sgn}\left(\sigma_{i}(k)\right) \sigma_{i}(k)-\varsigma_{i}\right) \\
& \quad-\beta_{i}(k) \operatorname{sgn}\left(\sigma_{i}(k)\right) \mid \\
& \quad=\mid\left(1-\alpha_{i}(k)+\gamma_{i}(k) \operatorname{sgn}\left(\sigma_{i}(k)\right)\right) \sigma_{i}(k)-\varsigma_{i} \\
& -\beta_{i}(k) \operatorname{sgn}\left(\sigma_{i}(k)\right)|=|\left(1-\epsilon_{i}\right) \sigma_{i}(k)-\varsigma_{i} \\
& -\beta_{i}(k) \operatorname{sgn}\left(\sigma_{i}(k)\right) \mid .
\end{aligned}
$$

Hence,

$$
\begin{aligned}
& \left|\sigma_{i}(k+1)\right| \\
& =\left|\left(1-\epsilon_{i}\right) \sigma_{i}(k)-\varsigma_{i}-\lambda_{i}\right|\left(1-\epsilon_{i}\right) \sigma_{i}(k)\left|\operatorname{sgn}\left(\sigma_{i}(k)\right)\right| \\
& =\left|\left(1-\lambda_{i}\right)\left(1-\epsilon_{i}\right) \sigma_{i}(k)-\varsigma_{i}\right| .
\end{aligned}
$$

This will ensure that $\left|\sigma_{i}(k+1)\right| \leq\left|\sigma_{i}(k)\right|$ is satisfied when the conditions below are met:

$$
\begin{gathered}
\sigma_{i}(k)<-\left[\frac{\varsigma_{i}}{\lambda_{i}+\epsilon_{i}-\lambda_{i} \epsilon_{i}}\right] \\
\text { or } \sigma_{i}(k)>-\left[\frac{\varsigma_{i}}{2-\left(\lambda_{i}+\epsilon_{i}-\lambda_{i} \epsilon_{i}\right)}\right] .
\end{gathered}
$$

In the case of $\sigma_{i}(k)<0$, it should be noted that, in order to ensure $\sigma_{i}(k)\left(\sigma_{i}(k+1)-\sigma_{i}(k)\right)<0$, there is another condition to be met; that is, $\alpha_{i}(k)>0$. Equation (20) stated that $\alpha_{i}(k)=$ $\gamma_{i}(k) \operatorname{sgn}\left(\sigma_{i}(k)\right)+\epsilon_{i}$, and $0<\epsilon_{i}<1$; therefore, in order to guarantee $\alpha_{i}(k)>0$, it is necessary to have

$$
\gamma_{i}(k)=\frac{M+N+L}{\left|\sigma_{i}(k)\right|}>-1 .
$$

So (34) implies that when $\sigma_{i}(k)<0$, the second condition to ensure the size of $\sigma_{i}(k)$ decreasing is

$$
\sigma_{i}(k)<-(M+N+L) \text {. }
$$

Since $\varsigma_{i}=M+N+L-Q_{i}(k)$ and $Q_{i}(k)<\left\|Q_{i}(k)\right\|<M+N+L$, this implies that

$$
0<\varsigma_{i}<2(M+N+L) .
$$

It can be concluded that $\sigma_{i}(k)$ exhibits a quasi-sliding mode with lower and upper bound $\left(\bar{U}_{l}\right.$ and $\bar{U}_{u}$, resp.) and the band is

$$
\begin{aligned}
& U_{l} \leq \sigma_{i}(k) \leq U_{u}, \\
& U_{l}>\bar{U}_{l}=\min \left(-\frac{2(M+N+L)}{l},-(M+N+L)\right), \\
& U_{u}<\bar{U}_{u}=\frac{2(M+N+L)}{2-l}
\end{aligned}
$$

with $l=\lambda_{i}+\epsilon_{i}-\lambda_{i} \epsilon_{i}$,

while $\sigma_{i}(k)=0$.

By substituting $\sigma_{i}(k)=0$ into $(20)$,

$$
\begin{aligned}
u_{i 1} & (k) \\
& =-\left(G_{i} B_{i}\right)^{-1}\left[G_{i} B_{i} \widehat{f}_{m i}(k)+G_{i} \widehat{f}_{u i}(k)-G_{i} x_{i}(k)\right] .
\end{aligned}
$$

Since, from (14),

$$
\begin{aligned}
\sigma_{i}(k)= & G_{i} x_{i}(k)-G_{i} x_{i}(0)-h_{i}(k), \\
u_{i 1}(k)= & -\widehat{f}_{m i}(k) \\
& -\left(G_{i} B_{i}\right)^{-1}\left(G_{i} \widehat{f}_{u i}(k)+h_{i}(k)-G_{i} x_{i}(0)\right),
\end{aligned}
$$

substituting (39) into (18) gives

$$
\begin{aligned}
\sigma_{i}(k+1)= & G_{i} B_{i}\left(f_{m i}(k)-\widehat{f}_{m i}(k)\right) \\
& +G_{i}\left(f_{u i}(k)-\widehat{f}_{u i}(k)\right) \\
& +G_{i} V_{i} \sum_{\substack{j=1 \\
j \neq i}}^{p} \emptyset_{j i} z_{j i}(k) .
\end{aligned}
$$

This concludes the proof for Theorem 2.

4.3. Overall System Stability. The overall system stability as the closed-loop performance while travelling along the sliding surface will be discussed in this section, that is, when $\sigma_{i}(k)=0$. Substituting (16) and (20) into (5), the closed-loop dynamic is derived below:

$$
\begin{aligned}
x_{i}(k+1)= & A_{i} x_{i}(k)+B_{i}\left(u_{i 0}(k)+u_{i 1}(k)+f_{m i}(k)\right) \\
& +f_{u i}(k)+V_{i} \sum_{\substack{j=1 \\
j \neq i}}^{p} \emptyset_{j i} z_{j i}(k) \\
= & A_{i} x_{i}(k)+B_{i}\left(f_{m i}(k)-\widehat{f}_{m i}(k)\right) \\
& +B_{i} u_{i 0}(k)+B_{i}\left(G_{i} B_{i}\right)^{-1} G_{i} x_{i}(0) \\
& +f_{u i}(k)-B_{i}\left(G_{i} B_{i}\right)^{-1} G_{i} \widehat{f}_{u i}(k) \\
& -B_{i}\left(G_{i} B_{i}\right)^{-1} h_{i}(k)+V_{i} \sum_{\substack{j=1 \\
j \neq i}}^{p} \emptyset_{j i} z_{j i}(k) .
\end{aligned}
$$


Since $h_{i}(k)=\sigma_{i}(k)-G_{i} x_{i}(k)+G_{i} x_{i}(0)$ and $u_{i 0}(k)=$ $-K_{i} x_{i}(k),(41)$ becomes

$$
\begin{aligned}
x_{i}(k+1)= & \left(A_{i}-B_{i} K_{i}\right) x_{i}(k) \\
& +B_{i}\left(f_{m i}(k)-\widehat{f}_{m i}(k)\right)+f_{u i}(k) \\
& -B_{i}\left(G_{i} B_{i}\right)^{-1} G_{i} \widehat{f}_{u i}(k) \\
& -B_{i}\left(G_{i} B_{i}\right)^{-1}\left(\sigma_{i}(k)-G_{i} x_{i}(k)\right) \\
& +V_{i} \sum_{\substack{j=1 \\
j \neq i}}^{p} \emptyset_{j i} z_{j i}(k) .
\end{aligned}
$$

Taking into account (11) and (40),

$$
\begin{aligned}
\sigma_{i}(k)= & G_{i} B_{i}\left(f_{m i}(k-1)-f_{m i}(k-2)\right) \\
& +G_{i}\left(f_{u i}(k-1)-f_{u i}(k-2)\right) \\
& +G_{i} V_{i} \sum_{\substack{j=1 \\
j \neq i}}^{p} \emptyset_{j i} z_{j i}(k-1) .
\end{aligned}
$$

Thus (42) becomes

$$
\begin{gathered}
x_{i}(k+1)=\left(A_{i}+B_{i}\left(G_{i} B_{i}\right)^{-1} G_{i}-B_{i} K_{i}\right) x_{i}(k) \\
+B_{i}\left[f_{m i}(k)-2 f_{m i}(k-1)+f_{m i}(k-2)\right] \\
+\left[f_{u i}(k)-2 B_{i}\left(G_{i} B_{i}\right)^{-1} G_{i} f_{u i}(k-1)\right. \\
\left.+B_{i}\left(G_{i} B_{i}\right)^{-1} G_{i} f_{u i}(k-2)\right]+\left[V_{i} \sum_{\substack{j=1 \\
j \neq i}}^{p} \emptyset_{j i} z_{j i}(k)\right. \\
\left.-B_{i}\left(G_{i} B_{i}\right)^{-1} G_{i} V_{i} \sum_{\substack{j=1 \\
j \neq i}}^{p} \emptyset_{j i} z_{j i}(k-1)\right] .
\end{gathered}
$$

Owing to the assumption that both $f_{m i}(k)$ and $f_{u i}(k)$ are bounded, it can be assumed that

$$
\begin{aligned}
& -W \leq B_{i}\left[f_{m i}(k)-2 f_{m i}(k-1)+f_{m i}(k-2)\right] \\
& +\left[f_{u i}(k)-2 B_{i}\left(G_{i} B_{i}\right)^{-1} G_{i} f_{u i}(k-1)\right. \\
& \left.+B_{i}\left(G_{i} B_{i}\right)^{-1} G_{i} f_{u i}(k-2)\right]+\left[V_{i} \sum_{\substack{j=1 \\
j \neq i}}^{p} \emptyset_{j i} z_{j i}(k)\right. \\
& \left.-B_{i}\left(G_{i} B_{i}\right)^{-1} G_{i} V_{i} \sum_{\substack{j=1 \\
j \neq i}}^{p} \emptyset_{j i} z_{j i}(k-1)\right]=w_{i}(k)
\end{aligned}
$$

$\leq W$, with $0<W<\infty$.
Then the closed-loop system dynamic can be represented by

$$
\begin{aligned}
x_{i}(k+1)= & \left(A_{i}+B_{i}\left(G_{i} B_{i}\right)^{-1} G_{i}-B_{i} K_{i}\right) x_{i}(k) \\
& +w_{i}(k) .
\end{aligned}
$$

The gain, $K$, must be selected so that $\bar{\rho}_{\max }<0$ with $\bar{\rho}_{\max }$ standing for the largest eigenvalue of $A_{i}+B_{i}\left(G_{i} B_{i}\right)^{-1} G_{i}-B_{i} K_{i}$.

Thus,

$$
\begin{aligned}
x_{i}^{T}(k) & {\left[x_{i}(k+1)-x_{i}(k)\right] } \\
= & x_{i}^{T}(k)\left(A_{i}+B_{i}\left(G_{i} B_{i}\right)^{-1} G_{i}-B_{i} K_{i}-I\right) x_{i}(k) \\
& +x_{i}^{T}(k) w_{i}(k) \\
\leq & \rho_{\max } x_{i}^{T}(k) x_{i}(k)+\left\|x_{i}^{T}(k) w_{i}(k)\right\| \\
\leq & \rho_{\max }\left\|x_{i}(k)\right\|^{2}+\left\|x_{i}^{T}(k) W\right\| .
\end{aligned}
$$

Since it is necessary to have $\rho_{\max }<0$ to ensure the stability of the system,

$$
\rho_{\max }<-\max \left(\frac{\left\|x_{i}^{T}(k) W\right\|}{\left\|x_{i}(k)\right\|^{2}}\right) .
$$

It can be seen from (47) that when the system is not affected by any uncertainty, that is, $W=0, \rho_{\max }<0$ is sufficient to ensure the system stability. When $W \neq 0$, the larger the uncertainty, $W$, is, the more negative $\rho_{\max }$ must be to guarantee the stability. This is due to the nature of discretetime system that $x_{i}(k)$ will never converge to zero but stays within a band about the origin.

\section{Examples and Simulation Results}

In this section, a simulation of large-scale discrete-time system with three interconnected subsystems is performed to illustrate the new control strategy. This example is taken from Park and Lee [6] and the dynamic equation of the subsystems can be written as

$$
\begin{aligned}
& x_{1}(k+1)=\left(\left[\begin{array}{ll}
0 & 1 \\
0 & 2
\end{array}\right]+\left[\begin{array}{cc}
0 & 0.4 \cos (k) \\
0.2 \sin (k) & 0
\end{array}\right]\right) \\
& \cdot x_{1}(k)+\left(\left[\begin{array}{l}
0 \\
1
\end{array}\right]+\left[\begin{array}{c}
0 \\
0.04 \sin (k)
\end{array}\right]\right) u_{1}(k) \\
& +\left(\left[\begin{array}{cc}
0 & 0.1 \\
0.1 & 0
\end{array}\right]+\left[\begin{array}{cc}
0 & 0 \\
0 & 0.05 \cos (k)
\end{array}\right]\right) x_{2}(k) \\
& +\left(\left[\begin{array}{cc}
0.05 & 0 \\
0 & 0.15
\end{array}\right]+\left[\begin{array}{cc}
0.01 \cos (k) & 0 \\
0 & 0.01 \sin (k)
\end{array}\right]\right) \\
& \cdot x_{3}(k),
\end{aligned}
$$




$$
\begin{aligned}
& x_{2}(k+1) \\
& =\left(\left[\begin{array}{cc}
0 & 1 \\
0.5 & -0.5
\end{array}\right]+\left[\begin{array}{cc}
0 & 0.04 \cos (k) \\
0.04 \sin (k) & 0
\end{array}\right]\right) \\
& \cdot x_{2}(k)+\left(\left[\begin{array}{l}
1 \\
1
\end{array}\right]+\left[\begin{array}{c}
0.04 \cos (k) \\
0
\end{array}\right]\right) u_{2}(k) \\
& +\left(\left[\begin{array}{cc}
0 & 0.2 \\
0.1 & 0
\end{array}\right]+\left[\begin{array}{cc}
0 & 0.04 \cos (k) \\
0 & 0
\end{array}\right]\right) x_{1}(k) \\
& +\left(\left[\begin{array}{cc}
0.15 & 0 \\
0 & 0.1
\end{array}\right]+\left[\begin{array}{cc}
0.01 \cos (k) & 0 \\
0 & 0.01 \sin (k)
\end{array}\right]\right) \\
& \text { - } x_{3}(k) \text {, } \\
& x_{3}(k+1) \\
& =\left(\left[\begin{array}{cc}
-0.9 & 0.5 \\
0 & 1
\end{array}\right]+\left[\begin{array}{cc}
0.1 \cos (k) & 0 \\
0 & 0.2 \sin (k)
\end{array}\right]\right) \\
& \cdot x_{3}(k)+\left(\left[\begin{array}{l}
1 \\
1
\end{array}\right]+\left[\begin{array}{c}
0 \\
0.05 \sin (k)
\end{array}\right]\right) u_{3}(k) \\
& +\left(\left[\begin{array}{cc}
0 & 0.05 \\
0.02 & 0.1
\end{array}\right]+\left[\begin{array}{cc}
0 & 0.1 \cos (k) \\
0.04 \sin (k) & 0
\end{array}\right]\right) \\
& \text { - } x_{1}(k) \\
& +\left(\left[\begin{array}{cc}
0 & 0.05 \\
0 & 0.1
\end{array}\right]+\left[\begin{array}{cc}
0 & 0.01 \cos (k) \\
0.01 \sin (k) & 0
\end{array}\right]\right) \\
& \text { - } x_{2}(k) \text {, }
\end{aligned}
$$

where

$$
\begin{aligned}
& x_{i}(k)=\left[\begin{array}{l}
x_{i 1}(k) \\
x_{i 2}(k)
\end{array}\right]^{T}, \\
& u_{i}(k)=\left[\begin{array}{l}
u_{i 1}(k) \\
u_{i 2}(k)
\end{array}\right]^{T}, \\
& i=1,2,3 .
\end{aligned}
$$

The initial conditions for this simulation are

$$
\begin{aligned}
& x_{1}(k)=\left[\begin{array}{ll}
1 & -0.5
\end{array}\right]^{T}, \\
& x_{2}(k)=\left[\begin{array}{ll}
-0.5 & -1.5
\end{array}\right]^{T}, \\
& x_{3}(k)=\left[\begin{array}{ll}
-1 & 0.5
\end{array}\right]^{T} .
\end{aligned}
$$

The value of $\epsilon_{i}(k)$ is chosen as $\epsilon_{1}=0.1, \epsilon_{2}=0.1$, and $\epsilon_{3}=0.1$. The value of $\lambda_{i}(k)$ is chosen as $\lambda_{1}=0.9, \lambda_{2}=0.2$, and $\lambda_{3}=0.3$. In this case, $G_{1}=\left[\begin{array}{ll}0 & 0.10\end{array}\right], G_{2}=\left[\begin{array}{ll}0.10 & 1\end{array}\right]$, and $G_{3}=\left[\begin{array}{ll}5 & 10\end{array}\right]$. The value of $K$ is obtained as $K_{1}=$ $\operatorname{place}\left(A_{1}+B_{1}\left(G_{1} B_{1}\right)^{-1} G_{1}, B_{1},\left[\begin{array}{ll}-0.9 & 0.9\end{array}\right]\right)=\left[\begin{array}{ll}-0.81 & 3.0\end{array}\right]$,
$K_{2}=\operatorname{place}\left(A_{2}+B_{2}\left(G_{2} B_{2}\right)^{-1} G_{2}, B_{2},\left[\begin{array}{ll}0.05 & 0.1\end{array}\right]\right)=[0.92$ $-0.57]$, and $K_{3}=\operatorname{place}\left(A_{3}+B_{3}\left(G_{3} B_{3}\right)^{-1} G_{3}, B_{3},\left[\begin{array}{ll}0.1 & 0.2\end{array}\right]\right)=$ [-0.45 1.25].

The sliding surfaces $\sigma_{1}(k), \sigma_{2}(k)$, and $\sigma_{3}(k)$ are chosen to be of the same parameter. Example below shows the equation of $\sigma_{1}(k), \sigma_{2}(k)$, and $\sigma_{3}(k)$ as implemented in the simulation:

$$
\begin{aligned}
& \sigma_{1}(k)=\left[\begin{array}{ll}
0 & 0.10
\end{array}\right] x_{1}(k)-\left[\begin{array}{ll}
0 & 0.10
\end{array}\right] x_{1}(0)+h_{1}(k \\
& -1)-\left(\left[\begin{array}{ll}
0 & 0.10
\end{array}\right]\left[\begin{array}{l}
0 \\
1
\end{array}\right] u_{10}(k-1)\right. \\
& \left.+\left[\begin{array}{ll}
0 & 0.10
\end{array}\right]\left[\begin{array}{ll}
0 & 1 \\
0 & 2
\end{array}\right] x_{1}(k-1)\right), \\
& \sigma_{2}(k)=\left[\begin{array}{ll}
0.10 & 1
\end{array}\right] x_{2}(k)-\left[\begin{array}{ll}
0.10 & 1
\end{array}\right] x_{2}(0)+h_{2}(k \\
& -1)-\left(\left[\begin{array}{ll}
0.10 & 1
\end{array}\right]\left[\begin{array}{l}
1 \\
1
\end{array}\right] u_{20}(k-1)\right. \\
& \left.+\left[\begin{array}{ll}
0.10 & 1
\end{array}\right]\left[\begin{array}{cc}
0 & 1 \\
0.5 & -0.5
\end{array}\right] x_{2}(k-1)\right), \\
& \sigma_{3}(k)=\left[\begin{array}{ll}
5 & 10
\end{array}\right] x_{3}(k)-\left[\begin{array}{ll}
5 & 10
\end{array}\right] x_{3}(0)+h_{2}(k-1) \\
& +\left(\left[\begin{array}{ll}
5 & 10
\end{array}\right]\left[\begin{array}{l}
1 \\
1
\end{array}\right] u_{30}(k-1)\right. \\
& \left.+\left[\begin{array}{ll}
5 & 10
\end{array}\right]\left[\begin{array}{cc}
-0.9 & 0.5 \\
0 & 1
\end{array}\right] x_{3}(k-1)\right),
\end{aligned}
$$

where

$$
\begin{array}{ll}
u_{10}(k)=-\left[\begin{array}{ll}
-0.81 & 3.0
\end{array}\right] x_{1}(k), & h_{1}(0)=0, \\
u_{20}(k)=-\left[\begin{array}{ll}
0.92 & -0.57
\end{array}\right] x_{2}(k), & h_{2}(0)=0, \\
u_{30}(k)=-\left[\begin{array}{ll}
-0.45 & 1.25
\end{array}\right] x_{3}(k), & h_{3}(0)=0 .
\end{array}
$$

$u_{11}(k)$ is the control signal for the first subsystem with the presence of disturbance and interconnection from the second and third subsystems:

$$
\begin{aligned}
& u_{11}(k)=-\left(\left[\begin{array}{ll}
0 & 0.10
\end{array}\right]\left[\begin{array}{c}
0 \\
10
\end{array}\right]\right)^{-1}\left[-\left[\begin{array}{ll}
0 & 0.10
\end{array}\right] x_{1}(k)\right. \\
& +\left[\begin{array}{ll}
0 & 0.10
\end{array}\right]\left[\begin{array}{l}
0 \\
1
\end{array}\right]\left(\left[\begin{array}{c}
0 \\
0.04 \sin (k)
\end{array}\right]\right) u_{1}(k-1) \\
& +\left[\begin{array}{ll}
0 & 0.10
\end{array}\right]\left[\begin{array}{cc}
0 & 0.4 \cos (k) \\
0.2 \sin (k) & 0
\end{array}\right] x_{1}(k) \\
& +\left[\begin{array}{ll}
0 & 0.10
\end{array}\right]\left[\begin{array}{cc}
0 & 0 \\
0 & 0.05 \cos (k)
\end{array}\right] x_{2}(k) \\
& +\left[\begin{array}{ll}
0 & 0.10
\end{array}\right]\left[\begin{array}{cc}
0.01 \cos (k) & 0 \\
0 & 0.01 \sin (k)
\end{array}\right] x_{3}(k) \\
& \left.+\alpha_{1}(k) \sigma_{1}(k)+\beta_{1}(k) \operatorname{sgn}\left(\sigma_{1}(k)\right)\right] \text {. }
\end{aligned}
$$


The same conditions applied to the second subsystem with the present of disturbance and interconnection effect. The control signals, $u_{21}(k)$ and $u_{31}(k)$, are given below:

$$
\begin{aligned}
& u_{21}(k)=-\left(\left[\begin{array}{ll}
0.10 & 1
\end{array}\right]\left[\begin{array}{l}
1 \\
1
\end{array}\right]\right)^{-1}\left[-\left[\begin{array}{ll}
0.10 & 1
\end{array}\right] x_{2}(k)\right. \\
& +\left[\begin{array}{ll}
0.10 & 1
\end{array}\right]\left[\begin{array}{l}
1 \\
1
\end{array}\right]\left(\left[\begin{array}{c}
0.04 \cos (k) \\
0
\end{array}\right]\right) u_{2}(k-1) \\
& +\left[\begin{array}{ll}
0.10 & 1
\end{array}\right]\left[\begin{array}{cc}
0 & 0.04 \cos (k) \\
0.04 \sin (k) & 0
\end{array}\right] x_{2}(k) \\
& +\left[\begin{array}{ll}
0.10 & 1
\end{array}\right]\left[\begin{array}{cc}
0 & 0.04 \cos (k) \\
0 & 0
\end{array}\right] x_{1}(k) \\
& +\left[\begin{array}{ll}
0.10 & 1
\end{array}\right]\left[\begin{array}{cc}
0.01 \cos (k) & 0 \\
0 & 0.01 \sin (k)
\end{array}\right] x_{3}(k) \\
& \left.+\alpha_{2}(k) \sigma_{2}(k)+\beta_{2}(k) \operatorname{sgn}\left(\sigma_{2}(k)\right)\right] \text {, } \\
& u_{31}(k)=-\left(\left[\begin{array}{ll}
5 & 10
\end{array}\right]\left[\begin{array}{l}
1 \\
1
\end{array}\right]\right)^{-1}\left[-\left[\begin{array}{ll}
5 & 10
\end{array}\right] x_{3}(k)\right. \\
& +\left[\begin{array}{ll}
5 & 10
\end{array}\right]\left[\begin{array}{l}
1 \\
1
\end{array}\right]\left(\left[\begin{array}{c}
0 \\
0.05 \sin (k)
\end{array}\right]\right) u_{3}(k-1) \\
& +\left[\begin{array}{ll}
5 & 10
\end{array}\right]\left[\begin{array}{cc}
0.1 \cos (k) & 0 \\
0 & 0.2 \sin (k)
\end{array}\right] x_{3}(k) \\
& +\left[\begin{array}{ll}
5 & 10
\end{array}\right]\left[\begin{array}{cc}
0 & 0.1 \cos (k) \\
0.04 \sin (k) & 0
\end{array}\right] x_{1}(k) \\
& +\left[\begin{array}{ll}
5 & 10
\end{array}\right]\left[\begin{array}{cc}
0 & 0.01 \cos (k) \\
0.01 \sin (k) & 0
\end{array}\right] x_{2}(k) \\
& \left.+\alpha_{3}(k) \sigma_{3}(k)+\beta_{3}(k) \operatorname{sgn}\left(\sigma_{3}(k)\right)\right] .
\end{aligned}
$$

The simulation has been done at a period of 50 seconds and the results are shown in Figures 1-7.

As shown in Figure 1 to Figure 3, the system trajectories of all the 3 subsystems of the discrete-time large-scale system under local control of discrete-time integral VSC are able to achieve stability and reached the desired conditions with disturbance being rejected. A comparison was made for the outputs of the systems with only the feedback control input, $u_{0}(k)$, without the VSC input, $u_{1}(k)$. It is clearly shown in Figures 4-6 that the system was unable to be controlled and unstable. Figure 7 showed that the sliding surface signals for all 3 subsystems achieved quasi-sliding mode with the discrete-time integral variable structure controller in place.

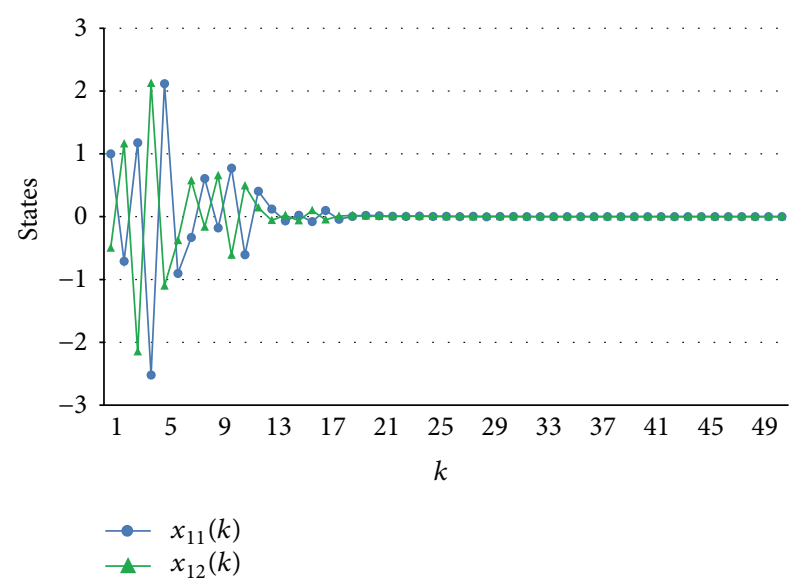

Figure 1: States respond of subsystem $1, x_{11}(k)$ and $x_{12}(k)$ under discrete-time integral VSC.

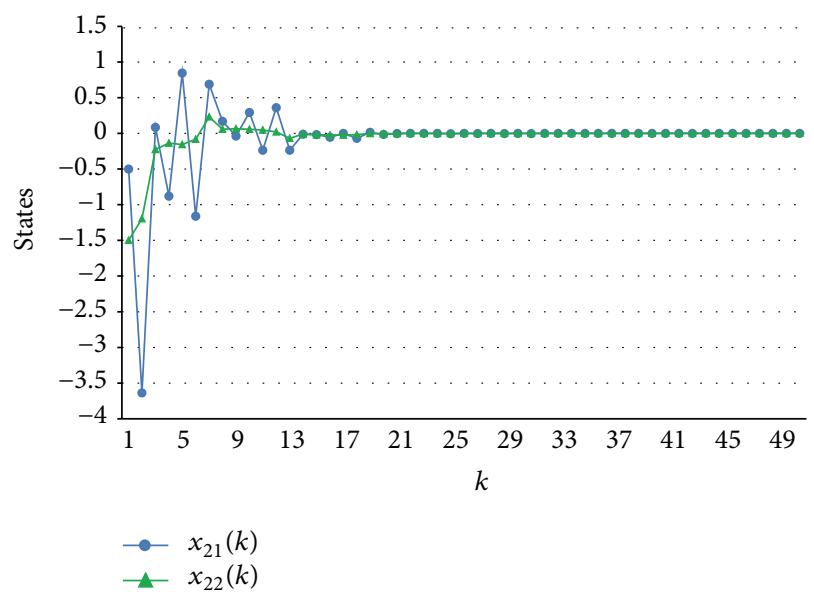

FIGURE 2: States respond of subsystem $2, x_{21}(k)$ and $x_{22}(k)$ under discrete-time integral VSC.

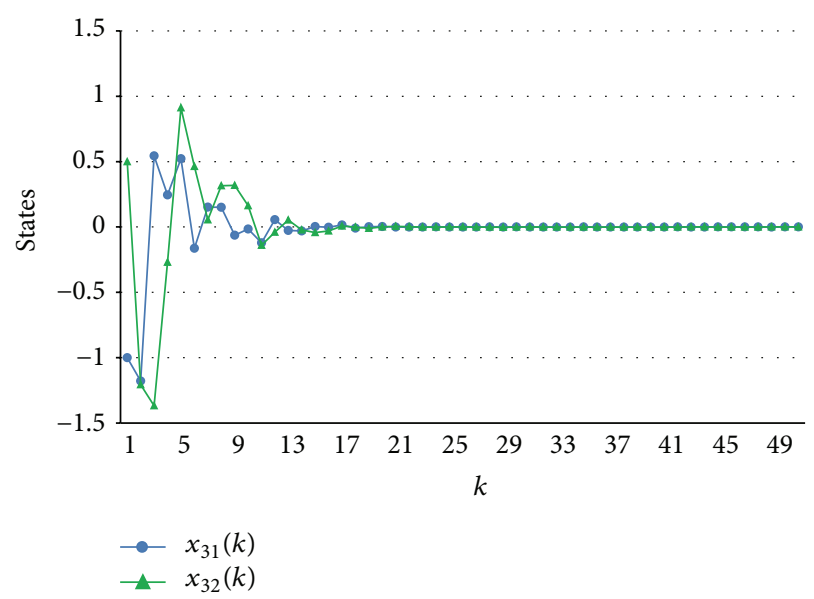

FIGURE 3: States respond of subsystem $3, x_{31}(k)$ and $x_{32}(k)$ under discrete-time integral VSC. 


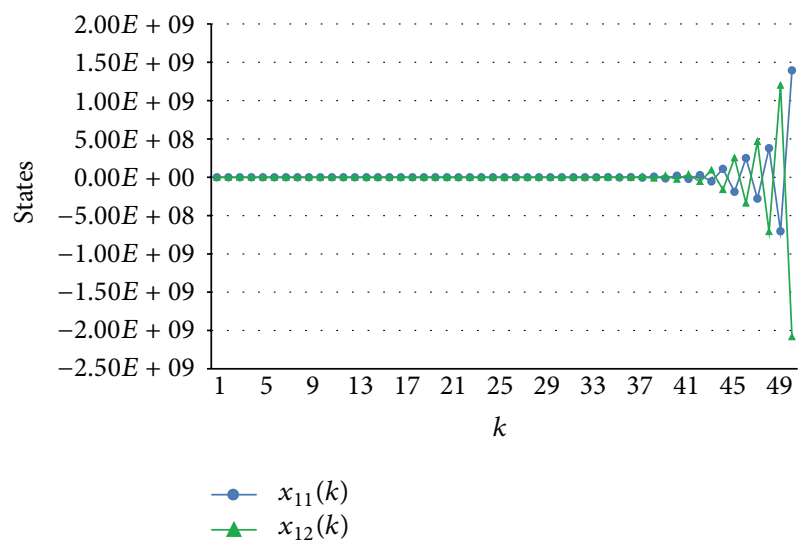

FIGURE 4: States respond of subsystem $1, x_{11}(k)$ and $x_{12}(k)$ under feedback control, without discrete-time integral VSC.

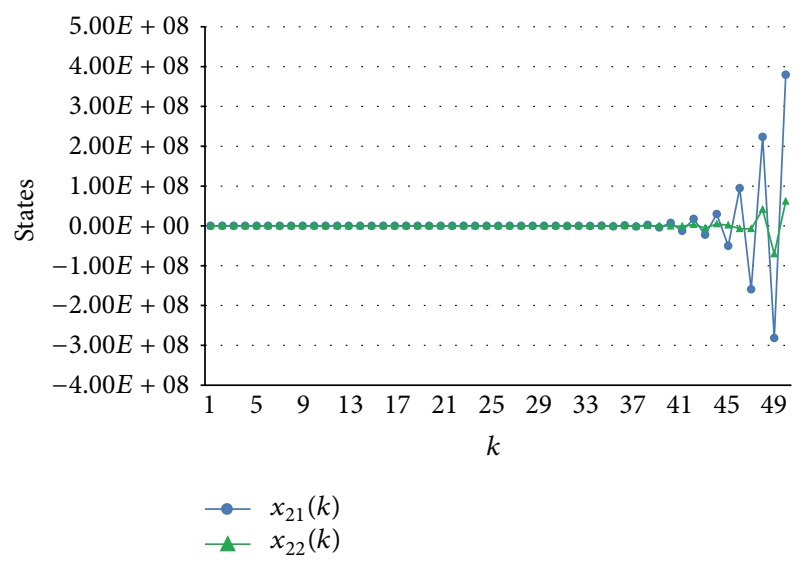

FIGURE 5: States respond of subsystem 2, $x_{21}(k)$ and $x_{22}(k)$ under feedback control, without discrete-time integral VSC.

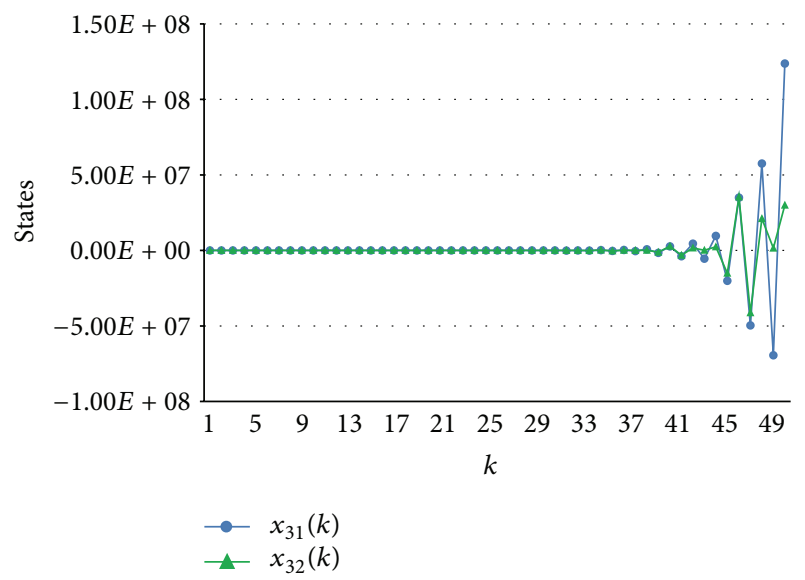

FIGURE 6: States respond of subsystem $3, x_{31}(k)$ and $x_{32}(k)$ under feedback control, without discrete-time integral VSC.

\section{Conclusion}

The control of large-scale discrete-time system with unmatched uncertainty by using integral variable structure control in local control method has been proposed in this paper. A new theorem has been presented and proved that,

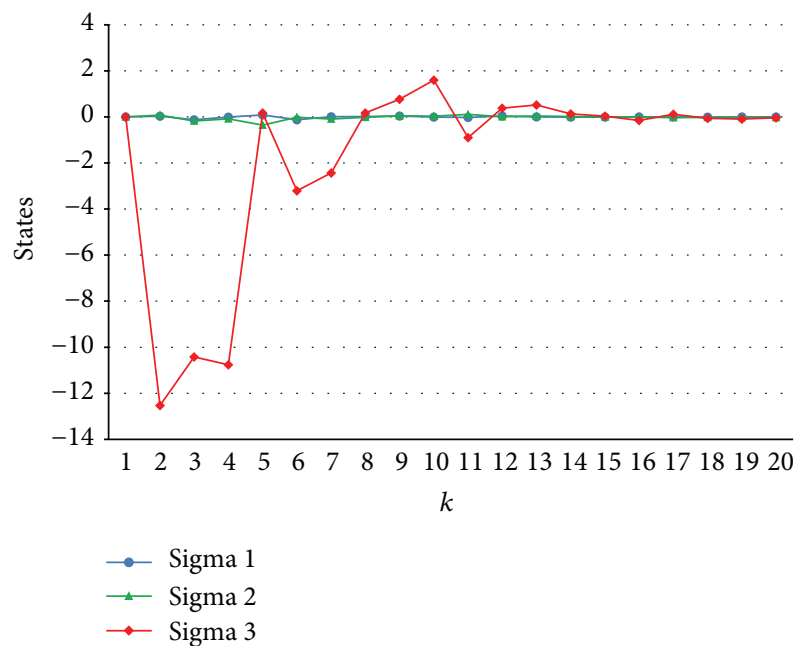

FIGURE 7: Sliding surface signal for subsystem $\left(\sigma_{1}(k)\right.$, sigma 1), subsystem $2\left(\sigma_{2}(k)\right.$, sigma 2$)$, and subsystem $3\left(\sigma_{3}(k)\right.$, sigma 3$)$.

with only the states feedback from the individual subsystem itself, the local controller is able to ensure that the system is achieving the quasi-sliding surface and remains there. The proposed local controller showed that the effect of interconnection in large-scale system is being handled well and the system stability is ensured. It is also shown that the effect of matched and unmatched uncertainty in the system is also being rejected. A discrete-time large-scale system comprised of 3 subsystems has been used to evaluate the performance of the local controller. It can be seen that the proposed controller is able to control the system to achieve the stability and desired value and also reduce the effect of disturbance as compared to the system without using VSC. As a conclusion, it can be concluded that the proposed discrete-time integral variable structure local controller has the advantage in controlling large-scale discrete-time system with matched and unmatched uncertainties.

\section{Conflict of Interests}

The authors declare that there is no conflict of interests regarding the publication of this paper.

\section{References}

[1] D. D. Siljak, Large-Scale Dynamic Systems: Stability and Structure, North-Holland, Amsterdam, The Netherlands, 1978.

[2] N. R. Sandell Jr., P. Varaiya, M. Athans, and M. G. Safonov, "Survey of decentralized control methods for large scale systems," IEEE Transactions on Automatic Control, vol. 23, no. 2, pp. 108128,1978

[3] Y. H. Chen, "Deterministic control of large-scale uncertain dynamical systems," Journal of the Franklin Institute, vol. 323, no. 2, pp. 135-144, 1987.

[4] G. Q. Li, K. Lee, and F. Gordon, "Decentralized control of discrete-time large-scale systems by dynamic programming," in Proceedings of the 21st IEEE Conference on Decision and Control, pp. 881-885, Orlando, Fla, USA, December 1982. 
[5] J. Lyou and Z. Bien, "Decentralized adaptive stabilization of a class of large-scale interconnected discrete systems," Journal of Dynamic Systems, Measurement and Control, vol. 107, no. 1, pp. 106-109, 1985.

[6] J. H. Park and S. G. Lee, "Robust decentralized stabilization of uncertain large-scale discrete-time systems," International Journal of Systems Science, vol. 33, no. 8, pp. 649-654, 2002.

[7] J. H. Park, H. Y. Jung, J. I. Park, and S. G. Lee, "Decentralized dynamic output feedback controller design for guaranteed cost stabilization of large-scale discrete-delay systems," Applied Mathematics and Computation, vol. 156, no. 2, pp. 307-320, 2004.

[8] O. Ou, Q. Hui, and H. Zhang, "Stability analysis and $\mathbf{H}_{\mathbf{\infty}}$ decentralized control for discrete-time nonlinear large-scale systems via fuzzy control approach," in Proceedings of the 6th International Conference on Fuzzy Systems and Knowledge Discovery (FSKD '09), vol. 4, pp. 166-170, IEEE, Tianjin, China, August 2009.

[9] Y. Dote and R. G. Hoft, "Microprocessor based sliding mode controller for DC motor drives," in Proceedings of the Industry Applications Society Annual Meeting, Cincinnati, Ohio, USA, September 1980.

[10] S. Z. Sarpturk, Y. Istefanopulos, and O. Kaynak, "On the stability of discrete-time sliding mode control systems," IEEE Transactions on Automatic Control, vol. 32, no. 10, pp. 930-932, 1987.

[11] D. Milosavljevic, "General conditions for the existence of a quasi-sliding mode on the switching hyperplane in discrete variable structure systems," Automation and Remote Control, vol. 46, pp. 307-314, 1985.

[12] K. Furuta, "Sliding mode control of a discrete system," Systems and Control Letters, vol. 14, no. 2, pp. 145-152, 1990.

[13] W. B. Gao, Y. Wang, and A. Homaifa, "Discrete-time variable structure control systems," IEEE Transactions on Industrial Electronics, vol. 42, no. 2, pp. 117-122, 1995.

[14] K. Abidi, J.-X. Xu, and X. Yu," "On the discrete-time integral sliding-mode control," IEEE Transactions on Automatic Control, vol. 52, no. 4, pp. 709-715, 2007.

[15] Z. Xi and T. Hesketh, "Discrete time integral sliding mode control for systems with matched and unmatched uncertainties," IET Control Theory and Applications, vol. 4, no. 5, pp. 889-896, 2010.

[16] A. F. Sheta, "Variable structure controller design for large-scale systems," in Proceedings of the IEEE International Workshop on Variable Structure Systems (VSS '96), pp. 228-231, December 1996.

[17] K. J. Hunt and T. A. Johansen, "Design and analysis of gainscheduled control using local controller networks," International Journal of Control, vol. 66, no. 5, pp. 619-651, 1997.

[18] G. Zhuansun and J. Xiong, "Local mode-dependent decentralised Ho control of uncertain Markovian jump large-scale systems," IET Control Theory and Applications, vol. 7, no. 7, pp. 1029-1038, 2013.

[19] W.-C. Su, S. V. Drakunov, and Ü. Özgüner, "An $O\left(T^{2}\right)$ boundary layer in sliding mode for sampled-data systems," IEEE Transactions on Automatic Control, vol. 45, no. 3, pp. 482-485, 2000.

[20] R. Morgan and U. Ozguner, "A decentralized variable structure control algorithm for robotic manipulators," IEEE Journal of Robotics and Automation, vol. 1, no. 1, pp. 57-65, 1985. 

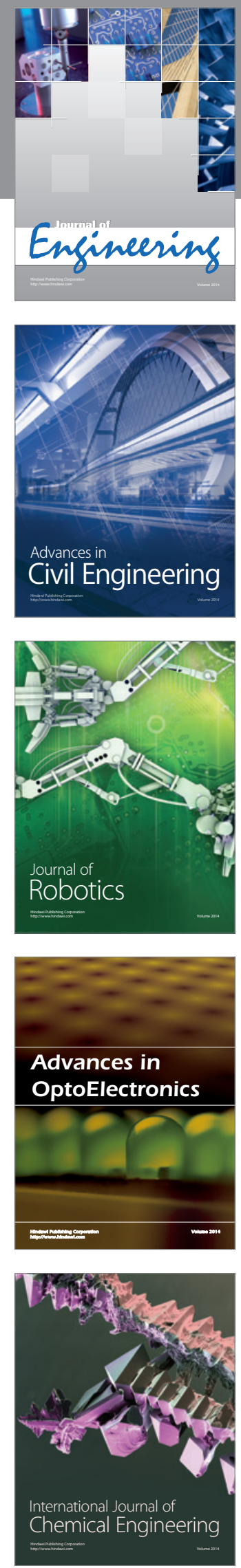

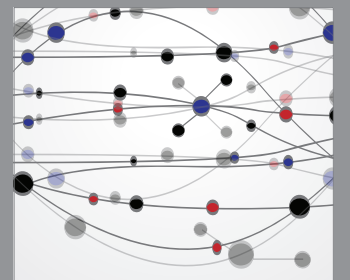

The Scientific World Journal
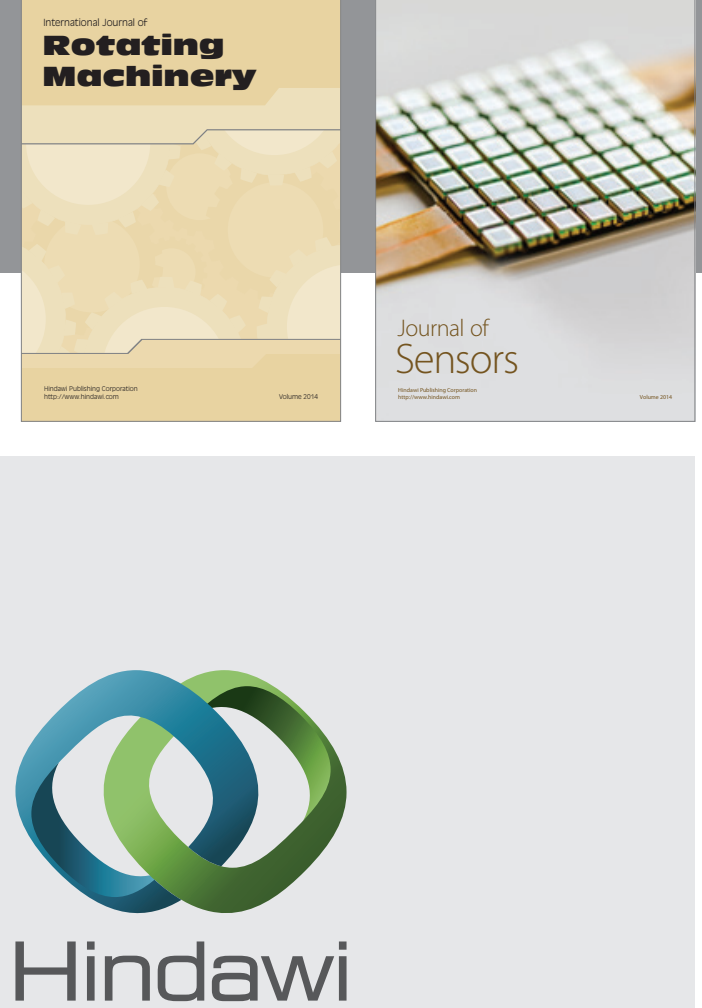

Submit your manuscripts at http://www.hindawi.com
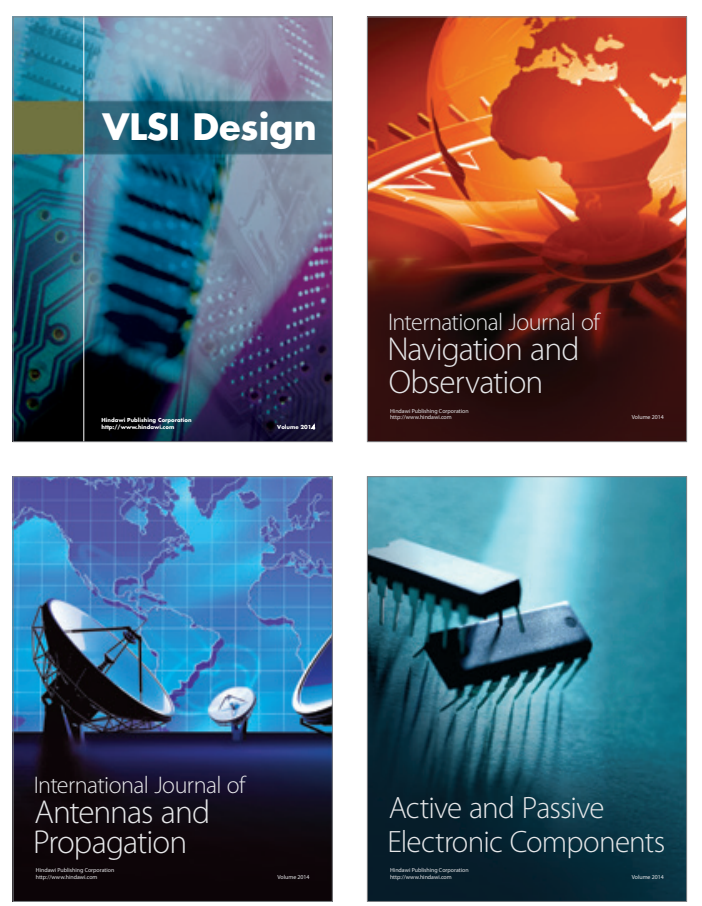
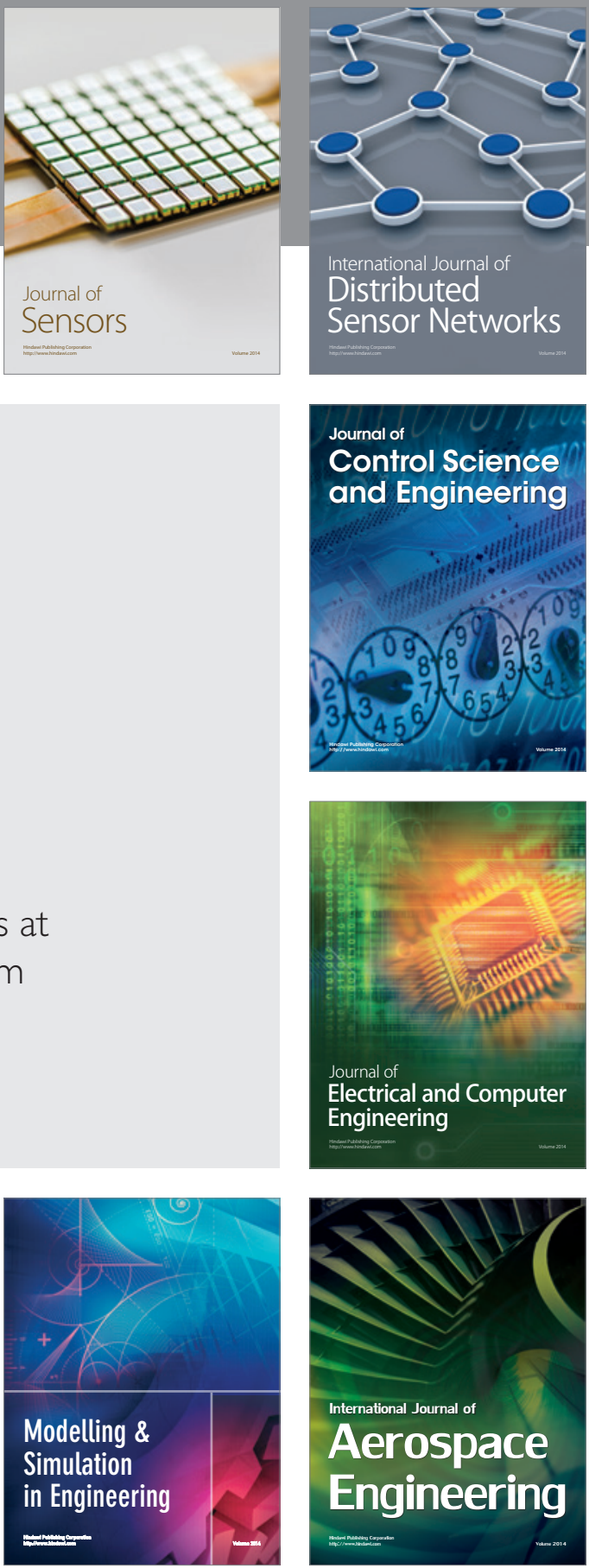

Journal of

Control Science

and Engineering
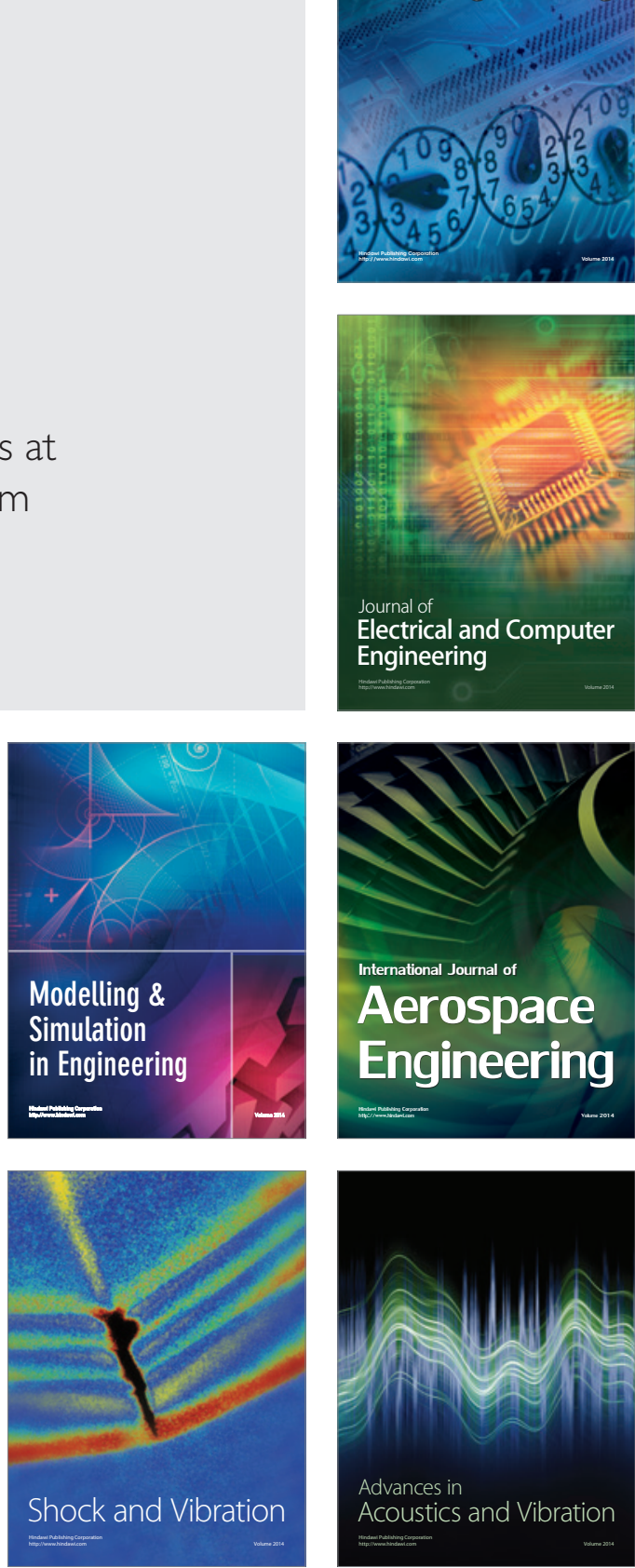